\title{
Competencia digital y tratamiento de la información en futuros maestros de Primaria
}

\section{Digital competence and information management in future primary school teachers}

\author{
Mario Grande-De-Prado ${ }^{1}$ \\ mgrap@unileon.es \\ Ruth Cañón-Rodríguez \\ rcanr@unileon.es \\ Isabel Cantón-Mayo \\ icanm@unileon.es \\ Universidad de León, España
}

\section{Resumen:}

Esta investigación tiene como objetivo analizar y conocer la autopercepción del alumnado que inicia el Grado de Maestro de Educación Primaria sobre su competencia para el tratamiento de la información digital. Esta capacidad para gestionar la información se ha convertido en nuestra sociedad en un eje fundamental y su desarrollo en los futuros formadores constituye un reto educativo relevante. Este estudio ha empleado una metodología descriptiva- interpretativa y ex post facto utilizando para la recogida de datos un cuestionario dirigido al alumnado del primer curso de Grado de Maestros de Educación Primaria de la Universidad de León. En términos generales, los resultados de la investigación señalan que el alumnado de la muestra se valora como muy competente en los aspectos digitales más simples y básicos, como la navegación en internet. En cam-

\begin{abstract}
:
This study aims to analyze the perception of beginner students in the Degree of Primary School Education about their competence to manage information. This ability to manage information has become a fundamental axis in present-day society and its development in teachers is an important educational challenge. This study has used a descriptive-interpretative ex post facto methodology. Data were collected through a questionnaire given to Primary School Degree students who had just started their degree at the University of León. The results indicate that the students regard themselves as highly competent in the most simple and basic digital aspects such as Internet browsing. In contrast, they seem unfamiliar with RSS feeds, social bookmarking sites or start webs. Some useful tools for students such as virtual
\end{abstract}

1 Dirección para correspondencia (correspondence address):

Mario Grande-de-Prado. Dpto. de Didáctica General, Específicas y Teoría de la Educación. Facultad de Educación. Universidad de León. Campus de Vegazana, s/n. 24071 León (España). 
bio, parece poco familiarizado con los agregadores RSS, los marcadores sociales o las webs de inicio; y la mayoría afirma no haber empleado herramientas tan útiles como los repositorios virtuales. Estas carencias en la gestión de la información, más allá de las habilidades más superficiales, son importantes para los futuros docentes y para las decisiones y medidas que se adopten en su formación inicial.

\section{Palabras clave:}

Competencia digital; Tratamiento de la información; TIC; Formación inicial; alumnado; educación superior; EEES. repositories have never been employed by a significant part of the sample.

These shortcomings in the management of information, beyond the most superficial skills, are important for future teachers and for decisions and actions taken in their initial training.

\section{Keywords:}

Digital Literacy; Information management; ICT; initial training; students; higher education; EHEA.

\section{Résumé:}

L`objectif de cette recherche a été la perception des étudiants universitaires du Grado d'enseignement primaire, sur la gestion de l'information et de leur propre culture digitale. Cette capacité pour gérer l'information est devenue dans notre société un axe clé et son développement chez les futurs maîtres est un important défi éducatif. Cette étude a utilisé une méthodologie descriptive-interpretative et ex post facto. Elle a utilisé pour la collecte de données un questionnaire adressé aux étudiants de première année du Grado d’Éducation Primaire (Université de León). Les résultats montrent que les futurs enseignants se sont auto- considérés compétents dans les aspects numériques les plus simples et basiques, comme la navigation sur Internet. Par contre, ils semblent peu familiarisés avec les flux RSS, les marqueurs sociaux ou les webs de commencement; et la majorité affirme n'avoir jamais employé d'outils comme les dépôts virtuels. Ces lacunes des futurs maîtres dans la gestion de linformation, au-delà des compétences les plus superficielles montrées, sont très importantes pour les futurs enseignants et pour la prise en compte dans leur formation initiale.

\section{Mots clés:}

Compétence digitale; Traitement de l'information; TIC; Formation initiale; maîtres; éducation supérieure; EEES.

Fecha de recepción: 4-4-2016

Fecha de aceptación: 27-6-2016

\section{Introducción}

Desde la aparición de las Tecnologías de la Información y Comunicación (TIC) éstas se han convertido en un eje fundamental en la sociedad actual (Vivancos, 2013; Cabero y Barroso, 2013; Vivancos, 2014), que sustituye el industrialismo por el informacionalismo (Castells, 2001). Son herramientas que propician el intercambio y la gestión de la información así como su posible elaboración y transformación en conocimiento, siendo las tecnologías digitales el principal recurso con el cual contamos para evolucionar (No- 
naka y Takeuchi, 1999; Castells, 2001), al permitirnos transformar nuestro pensamiento y nuestra relación con la información (Gros, 2008).

Por tanto, en la sociedad actual lo importante ya no es dominar determinados conocimientos, sino qué saber y cómo acceder al conocimiento en el momento requerido (Cabero y Barroso 2013; Cantón y Pino, 2014). El conocimiento esta irremediablemente unido a nuestra capacidad para percibir los cambios continuos que se suceden y adaptarnos a ellos, ya que nuestra competencia para aprender a aprender es fundamental en el entorno actual (Cantón, 2007).

En base a ello, la capacidad para emplear dichas tecnologías de manera eficaz, lo que podemos entender como una definición rudimentaria de competencia digital, debe capacitarnos para conseguir metas en lo laboral, en lo educativo, en el ocio y facilitar la participación ciudadana (Ferrari, 2013; INTEF, 2014). Por tanto, el uso educativo de las herramientas para acceder a cualquier tipo de información, procesarla y transformarla, debe tenerse en cuenta en la formación inicial de los maestros, siendo evidente la existencia de nuevas necesidades a las que debemos encontrar respuesta (Cantón y Baelo, 2011; Aguaded, 2012; 2014; Area, Borrás y San Nicolás, 2014; Cabero y Barroso, 2015; De Pablos, 2015; Roblizo y Cózar, 2015; Sevillano y Vázquez, 2015; Zafra, 2015).

\section{Marco teórico}

Actualmente, la formación del profesorado se encuentra ante el reto y la necesidad de vincular las TIC a las prácticas pedagógicas (Comisión de las Comunidades Europeas, 2002, 2013; Llorente Cejudo, 2008; Área y Guarro, 2012. Álvarez y Gisbert, 2015), por lo que es necesario que la formación inicial de los futuros maestros se encamine hacia su logro.

A pesar de que existen prácticas de calidad vinculadas al uso de las TIC y cada vez un mayor interés en el uso educativo de las herramientas TIC (Adell y Castañeda, 2012; Pérez y Delgado, 2012; Cabero y Barroso, 2013; Álvarez y Gisbert, 2015), especialmente en la aplicación de microblogging, con servicios como Twitter o Edmondo (Vazquez, 2013; Abella y Delgado, 2015); sigue existiendo una gran preocupación por resolver las diferentes dificultades en competencia digital de los futuros maestros (Cabero y Barroso, 2013). 
Diversas investigaciones, nacionales (Espuny, González y Gisbert, 2010; Roig y Pascual, 2012; Pérez y Vílchez, 2012; o Cabezas, Casillas y Pinto, 2014) e internacionales (Burnett, 2011; Duncan-Howell, 2012; Chen, Zhou, Tan y Wong, 2013; y Rokebes y Krumsvik, 2014) sobre la competencia digital de los futuros maestros, señalan las dificultades que éstos tienen para asimilar el impacto de las TIC en la Sociedad y emplearlas en el proceso educativo.

Ante esta situación han ido apareciendo diferentes modelos de competencia digital (MEC, 2007; Marques, 2009), de los cuáles el modelo DIGCOMP, dentro del contexto del Espacio Europeo de Educación Superior (EEES) está cobrando especial relevancia (Ferrari, 2013).

Este modelo, adaptado en nuestro país (INTEF, 2014), desglosa la competencia digital en 21 subcompetencias, organizadas a su vez en cinco áreas o dimensiones (Ferrari, 2013; INTEF, 2014), muchas de ellas relacionadas e interconectadas entre sí: Información, Comunicación, Creación de contenidos, Seguridad y Resolución de Problemas.

\section{COMPETENCIA DIGITAL}

\begin{tabular}{|c|c|c|c|c|}
\hline INFORMACIÓN & COMUNICACIÓN & $\begin{array}{l}\text { CREACIÓN DE } \\
\text { CONTENIDOS }\end{array}$ & SEGURIDAD & $\begin{array}{l}\text { RESOLUCIÓN DE } \\
\text { PROBLEMAS }\end{array}$ \\
\hline $\begin{array}{l}\text { Navegación, } \\
\text { búsqueda y } \\
\text { filtrado de infor- } \\
\text { mación. } \\
\text { Evaluación de } \\
\text { información. } \\
\text { Almacenamiento } \\
\text { y recuperación } \\
\text { de información. }\end{array}$ & $\begin{array}{l}\text { Interacción a través } \\
\text { de medios digitales } \\
\text { Compartir informa- } \\
\text { ción y contenidos } \\
\text { Participación ciuda- } \\
\text { dana en línea } \\
\text { Colaboración } \\
\text { mediante medios } \\
\text { digitales } \\
\text { Netiqueta } \\
\text { Gestión de la iden- } \\
\text { tidad }\end{array}$ & $\begin{array}{l}\text { Desarrollo de } \\
\text { contenidos } \\
\text { Integración y } \\
\text { reestructura- } \\
\text { ción } \\
\text { Derechos de } \\
\text { autor y licen- } \\
\text { cias } \\
\text { Programación. }\end{array}$ & $\begin{array}{l}\text { Protección de } \\
\text { dispositivos } \\
\text { Protección de } \\
\text { datos persona- } \\
\text { les e identidad } \\
\text { digital } \\
\text { Protección de } \\
\text { la salud } \\
\text { Protección del } \\
\text { entorno. }\end{array}$ & $\begin{array}{l}\text { Resolución de pro- } \\
\text { blemas técnicos } \\
\text { Identificación de } \\
\text { necesidades y } \\
\text { respuestas tecno- } \\
\text { lógicas } \\
\text { Innovación y uso } \\
\text { de la tecnología de } \\
\text { forma creativa } \\
\text { Identificación de } \\
\text { lagunas en la com- } \\
\text { petencia digital. }\end{array}$ \\
\hline
\end{tabular}

Figura 1. Competencias Digitales Docentes DIGCOMP (elaboración propia basada en Ferrari, 2013).

En base a lo expuesto y dada la relevancia de la formación inicial y los retos que las Facultades de Educación tienen que afrontar, se ha planteado una investigación con la intención de conocer la autopercepción del alumnado del Grado de Educación Primaria sobre el Tratamiento de la Información dentro la competencia digital, tomando como referencia 
el citado modelo DIGCOMP, en el que Ferrari (2013), define la Información como la capacidad de los docentes para "Identificar, localizar, obtener, almacenar, organizar y analizar información digital, evaluando su finalidad y relevancia para las tareas docentes" (INTEF, 2014:47). Lo que conlleva que los futuros maestros sean competentes en búsqueda, selección, almacenamiento y evaluación de la información.

Tomando como referencia el modelo DIGCOMP y las aportaciones sobre competencia digital de Fernández, Cebreiro, y Fernández (2010), en esta investigación optamos por valorar las competencias agrupándolas en torno dos polos: la navegación por internet (búsqueda y acceso a la información), y a la gestión de la información.

Para concluir el estado de la cuestión reflejamos algunos datos aportados por otras investigaciones sobre sobre el tratamiento de la información de jóvenes y futuros maestros. Los estudios realizados en alumnos de Secundaria y Bachillerado mediante el cuestionario COTASEBA (Cabero y Llorente, 2006; Pons y Salinas, 2008; Fernández, Cebreiro y Fernández, 2009; 2011) muestran al alumnado competente en la navegación por Internet, pero no en los procesos de búsqueda y gestión de información. Resultados similares aparecen en los estudios realizados sobre estudiantes universitarios en el Grado de Maestro o Magisterio. Espuny, González y Gisbert (2010) encuentran que están familiarizados con la navegación pero presentan carencias en búsqueda de información. Roig y Pascual (2012) observan dificultades en aspectos complejos del tratamiento de la información. Pérez y Vilches (2012) indican que el alumnado es incapaz de asimilar las posibilidades de las TIC y su impacto en la sociedad y en la educación. Por último, Cabezas, Casillas y Pinto (2014) aportan que el alumno se describe como competente en el manejo de la información, aspecto que las autoras señalan como llamativo y contradictorio, siendo revelador en ese sentido que no se sientan capacitados en el manejo de bases de datos. Todo esto parece indicarnos que los futuros maestros realizan actividades sencillas como navegación por internet, pero no son hábiles en el manejo de la información, según los estudios citados. 


\section{Marco empírico}

\subsection{Objetivo}

Dada la relevancia que concedemos a la capacidad para gestionar la información tanto en la sociedad como en la formación de los futuros maestros, la investigación se ha articulado en torno al objetivo de analizar y conocer la autopercepción que el alumnado del Grado de Maestro de Educación Primaria de la Universidad de León tiene del tratamiento de la información dentro de la competencia digital.

\subsection{Metodología}

En esta investigación se ha optado por la metodología no experimental de tipo descriptivo-interpetativo y ex-postfacto (McMillan y Schumacher, 2005; Hernández, Colás y Buendía, 2010; Sevillano y Vázquez, 2015), que puede dar paso a un proceso de mejora.

\subsection{Muestra}

Para obtener la muestra se ha partido de un universo-población conformado por todo el alumnado del primer curso del Grado de Maestro de Educación Primaria de una única Facultad durante los cursos 11/12, $12 / 13$. $13 / 14$ y $14 / 15$, con un total de alumnos de 492. La muestra recogida fue de 329 alumnos. Esto nos ofrece un nivel de confianza del 99\% y un porcentaje de error del $4.09 \%$.

\subsection{Instrumento}

Dada la amplitud de la muestra se ha optado por utilizar un único instrumento, el cuestionario para recoger la información sobre las percepciones que los futuros maestros, que hemos denominado COTATI (Cuestionario de Competencias tecnológicas de los alumnos en Tratamiento de la Información).

Para su construcción hemos tomado como referencia inicial el Cuestionario COTASEBA (Cabero y Llorente, 2006) del que seleccionamos 24 de los 30 ítems que conforman el instrumento, basándonos en la descripción de la dimensión de información del DIGCCOMP (INTEF, 2014). 
Se realizó una validación de constructo con cinco expertos que tuvieron en cuenta la univocidad, pertinencia y relevancia de los items, y se realizó la medición de fiabilidad (Alfa de Cronbach y las Dos mitades de Guttman) obteniéndose valores de .912 y .825, respectivamente. Finalmente el cuestionario utilizado quedó compuesto por 30 ítems, de los cuáles cinco son cualitativos y los otros 25 son ítems en forma de escala Likert con 10 posibilidades de respuesta (0-10), donde los valores 0-4 se consideran negativos y los valores entre 5 y 10 como positivos, siguiendo las propuestas realizadas en el COTASEBA.

Algunos de estos ítems hacen referencia a la competencia digital propiamente dicha y otros a la frecuencia de uso (estos últimos son ítems no presentes en el COTASEBA y que sí están en nuestro instrumento). En este último caso los valores negativos 0-4 hacen referencia al uso una vez al mes o menos (los valores 0 y 1 expresan que nunca se ha empleado) y los valores entre 5 y 10 indican un uso de ese recurso al menos varias veces al mes. Estos ítems han quedado distribuidos en dos subdimensiones inspirándonos en las competencias del modelo DIGCOMP (Ferrari, 2013), que considera la presencia de tres subcompetencias, y las aportaciones de Fernández, Cebreiro, y Fernández (2010) sobre la competencia digital empleando el COTASEBA. Estas subdimensiones que empleamos son Navegación por internet y Gestión de la información, a las que les corresponden 8 y 17 ítems (ver Tabla 1).

Recogida la información, procedimos a analizar los datos obtenidos, empleando para ello el paquete estadístico SPSS (Statistical Package Social Science), versión 21.0.

\section{Resultados y discusión}

\subsection{Características generales}

De los 329 sujetos que forman la muestra, el $69.9 \%$ son mujeres y el $30.1 \%$ son hombres, que han cursado el primer año de del Grado de Maestro en Educación Primaria en el curso 2014/2015 (32,2\%) en $2012 / 2013$ y $2013 / 2014$ (23,7\%), y en el curso $2011 / 2012$ (20,4\%). Todos ellos alumnos de primer año de Grado en Educación Primaria

Son sujetos que en su mayoría $(89,1 \%)$ consideran que Internet tiene un gran peso en su vida diaria. 


\subsection{Análisis factorial}

De las variables asociadas a la información resulta una estructura con cuatro factores que explican el $58.452 \%$ de la varianza total.

- Factor I: Este factor, denominado Manejo de la información (I1), explica el $26.405 \%$ de la varianza, e incluye los ítems $(14 ; 15 ; 16$; $17 ; 18 ; 19 ; 20 ; 21 ; 22 ; 23 ; 24 ; 25$ y 26 ) referidos al tratamiento de la información, pudiendo manifestarse en subdimensiones tanto de búsqueda, como de evaluación y organización de la información.

- Factor II: Este factor, al que hemos nombrado Navegación Básica (I2), explica el $17.719 \%$ de la varianza y se vincula con los usos más comunes de navegación y búsqueda de información por internet (ítems $6 ; 7 ; 8 ; 9 ; 10 ; 11 ; 12$ y 13), sobresaliendo en puntuación los ítems referidos a la descarga de internet y al uso de diferentes buscadores.

- Factor III: Este factor, denominado Frecuencia de uso de herramientas 2.0 (I3), explica el $9.296 \%$ de la varianza y engloba los ítems $(27 ; 28$ y 29$)$ referidos a la frecuencia de usos de algunas opciones de la Web 2.0, lo cual está relacionado con lo hábiles que serán con estas herramientas.

- Factor IV: El último factor, el de Enciclopedias Online (14), explica el $5.032 \%$ de la varianza y se vincula con la frecuencia de uso de las Enciclopedias Online (ítem 30).

Tabla 1. Relación de factores del análisis factorial de la dimensión de Información con las subdimensiones del COTATI.

\begin{tabular}{|c|c|c|c|c|}
\hline Abreviatura & Subdimensiones & $\begin{array}{l}\text { Factor (Análisis } \\
\text { Factorial) }\end{array}$ & Ítems & $\begin{array}{c}\text { Ítems por } \\
\text { Subdimensión } \\
\end{array}$ \\
\hline INF-A1 & Navegación & $\begin{array}{c}\text { Navegación Básica } \\
\text { (I2) }\end{array}$ & $\begin{array}{c}6,7,8,9,10 \\
11,12,13\end{array}$ & 8 \\
\hline \multirow[t]{3}{*}{ INF-A2BC } & \multirow{3}{*}{$\begin{array}{l}\text { Gestión de la } \\
\text { información }\end{array}$} & $\begin{array}{l}\text { Manejo de la } \\
\text { información (I1) }\end{array}$ & $\begin{array}{c}14 ; 15 ; 16 ; \\
17 ; 18 ; 19 ; \\
20 ; 21 ; 22 ; \\
23 ; 24 ; 25 \\
\quad \text { y } 26 \\
\end{array}$ & \multirow[t]{3}{*}{17} \\
\hline & & $\begin{array}{l}\text { Frecuencia de uso } \\
\text { de herramientas (I3) }\end{array}$ & $27 ; 28$ y 29 & \\
\hline & & $\begin{array}{l}\text { Enciclopedias } \\
\text { Online (I4) }\end{array}$ & 30 & \\
\hline
\end{tabular}




\subsection{Resultados por subdimensiones}

\subsubsection{Subdimensión Navegación Básica INF-A1}

En esta subdimensión (INF-A1), se engloban los ítems que hacen referencia a la Navegación básica (I2). Se obtienen medias superiores a 5 en todos los ítems a excepción del ítem 12, referido a la familiaridad con páginas de almacenamiento virtual (4.73), cual nos lleva a señalar que el alumnado se siente como mínimo moderadamente competente para realizar todas las demás habilidades de esta subdimensión.

Tabla 2. Medidas de tendencia central de los ítems del factor I2/Subdimensión INF-A1.

\begin{tabular}{ccccccccc}
\hline ITEM & Media & Mediana & Moda & $\begin{array}{c}\text { Desv. } \\
\text { Típica }\end{array}$ & $\begin{array}{c}\text { Perc. } \\
\mathbf{2 5}\end{array}$ & $\begin{array}{c}\text { Perc. } \\
\mathbf{5 0}\end{array}$ & $\begin{array}{c}\text { Perc. } \\
\mathbf{7 5}\end{array}$ \\
\hline $\begin{array}{c}\text { 6. Navegación; } \\
\text { navegadores }\end{array}$ & 7.46 & 8.00 & 10 & 2,186 & 6.00 & 8.00 & 9.00 \\
\hline $\begin{array}{c}\text { 7. Navegación; uso de } \\
\text { enlaces }\end{array}$ & 7.22 & 8.00 & 10 & 2.284 & 6.00 & 8.00 & 9.00 \\
\hline $\begin{array}{c}\text { 8. Descargar de } \\
\text { internet }\end{array}$ & 7.77 & 8.00 & 9 & 2.037 & 7.00 & 8.00 & 9.00 \\
\hline $\begin{array}{c}\text { 9. Uso de diferentes } \\
\text { buscadores }\end{array}$ & 8.06 & 8.00 & 10 & 1.820 & 7.00 & 8.00 & 10.00 \\
\hline $\begin{array}{c}\text { 10. Búsqueda } \\
\text { avanzada }\end{array}$ & 6.08 & 6.00 & 5 & 2.508 & 5.00 & 6.00 & 8.00 \\
\hline 11. Favoritos & 6.69 & 7.00 & 6 & 2.328 & 5.00 & 7.00 & 8.00 \\
\hline $\begin{array}{c}\text { 12. Familiaridad con } \\
\text { almacenamiento } \\
\text { virtual }\end{array}$ & 4.73 & 5 & 5 & 2.822 & 2 & 5 & 7 \\
\hline 13. Buscadores Web & $\mathbf{8 . 8 1}$ & $\mathbf{1 0}$ & $\mathbf{1 0}$ & $\mathbf{1 . 9 1 5}$ & $\mathbf{8}$ & $\mathbf{1 0}$ & $\mathbf{1 0}$ \\
\hline
\end{tabular}

Los ítems mejor puntuados, nos indican que el alumnado participante considera que utiliza casi a diario diferentes buscadores web (8.81), y que se siente bastante competente para usar diferentes buscadores de internet (8.06), la realización de diferentes descargas en internet (7.77), y la navegación por internet con diferentes navegadores (7.46) y mediante distintos, links, enlaces o hipervínculos (7.22).

Por el contrario, el alumnado encuestado ha señalado que no se considera sufrientemente competente (4.73) con las páginas de almacenamiento virtual (ítem 12), aunque por un escaso margen ya que otras medidas de tendencia central (mediana y moda) tienen el valor 5, lo cual 
nos hace pensar junto con la desviación típica (2.822) que existe una cierta heterogeneidad en las respuestas.

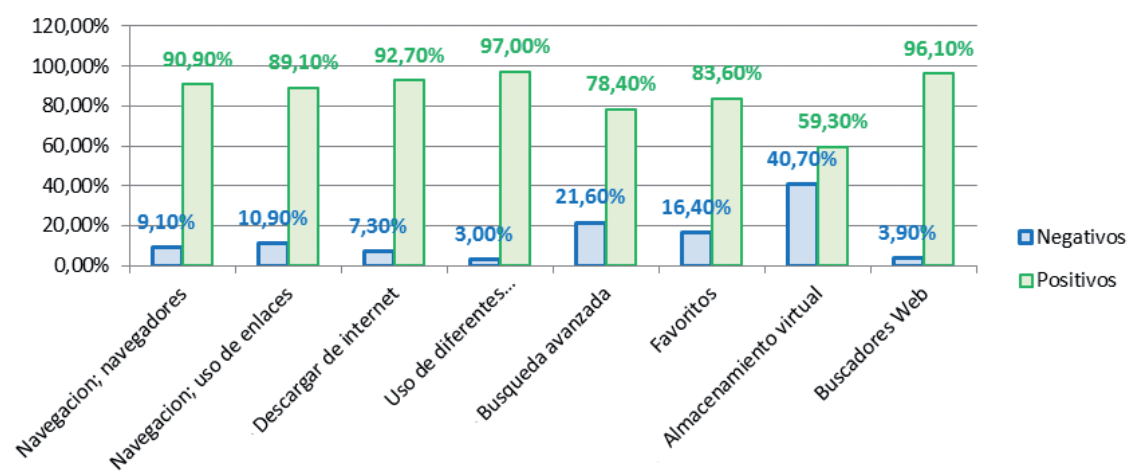

Figura 2. Frecuencia de respuestas negativas y positivas de los ítems del factor I2 / Subdimensión INF-A1.

En la Figura 2, se muestra de manera global un alto índice de valoraciones positivas agrupadas (de 5 a 10) en las que destaca el ítem $n^{\circ}$ $9(97 \%)$, referido a la utilización de diferentes buscadores de internet mientras que desde la visión contraria, la familiaridad con las páginas de almacenamiento virtual (ítem 12), es el que presenta la valoración negativa más alta del indicador $(40.7 \%)$, hecho situándose un $10.0 \%$ en el valor cero, complementado por una moda de 5 .

En base a las puntuaciones obtenidas, podemos afirmar que la mayoría del alumnado encuestado se siente muy competente en cuestiones relacionadas con aspectos básicos de la navegación por internet, en la que los algunos ítems $(6,7,8,9$ y 13) presentan perfiles que demuestran una tendencia marcadamente positiva, con frecuencias altas a partir de valores superiores a 7, de entre los cuáles en el ítem 13 destaca especialmente la moda en valor extremo 10.

En cambio, aunque el alumnado también considere que domina los aspectos relacionados con actividades menos habituales en la navegación pero sin un nivel de dificultad reseñable, como son la búsqueda avanzada en diferentes buscadores de Internet (ítem 10), la organización de la información recogida de Internet, agregando las páginas a favoritos, y clasificarlas en subcarpetas bajo algún criterio de ordenación (ítem 11) así como la familiaridad con las páginas de almacenamiento virtual (ítem 12), mostrando tendencias positivas, pero si bien estas aparecen más 
repartidas entre las frecuencias positivas del ítem 11, y en el caso del 10 parecen más atraídas por la moda (situada en 5), encontrándose fundamentalmente entre 5 y 8 , y en el ítem 12 aparecen también frecuencias altas entre 5 y 8 pero con una presencia importante de frecuencias en los valores negativos (con un $10 \%$ en el valor 0 ).

\subsubsection{Subdimensión Gestión de LA INFORMACIÓN INF-A2BC}

En esta Subdimensión, se encuentran recogidos tres factores resultantes del análisis factorial. El primero de ellos, hace referencia a manejo de la información (I1) y agrupa los ítems $14 ; 15 ; 16 ; 17 ; 19 ; 20 ; 21 ; 22 ; 23$; $24 ; 25$ y 26 . El segundo de ellos, frecuencia de uso de las herramientas 2.0 engloba los ítems 27, 28 y 29, y por último el factor referido a las enciclopedias online que únicamente se corresponde con el ítem 30.

Observando las medidas de tendencia central, hemos obtenido medias superiores a 5 en tan sólo 8 ítems de los 17, siendo siete de ellos parte del Factor 11, manejo de la información. De todos ellos, el valor más alto es el referido a la frecuencia de uso de las Enciclopedias online (6.66) y en cambio el más bajo el ítem 29, referido a la frecuencia de uso de marcadores sociales (0.77).

En general no se observan medidas de tendencia central altas, presentándose algunos ítems en los que estas aparecen significativamente por debajo de las demás, como el ítem 16 sobre el uso de protocolos de transferencia de archivos (3.45), el ítem 27 sobre la frecuencia de uso de webs de inicio (3.61) el ítem 28 sobre la frecuencia de uso: agregadores RSS $(1,70)$ y el ítem 29 sobre la frecuencia de uso de marcadores sociales, ya citado anteriormente.

Tabla 3. Medidas de tendencia central de los ítems Subdimensión INF-A2BC.

\begin{tabular}{cccccccc}
\hline ITEM & \multicolumn{1}{c}{ Media Mediana Moda } & Desv. Perc. Perc. Perc. \\
& & & & Tíca & $\mathbf{2 5}$ & $\mathbf{5 0}$ & $\mathbf{7 5}$ \\
\hline $\begin{array}{c}\text { 14. Consultas en bases de } \\
\text { datos }\end{array}$ & 4.39 & 5.00 & 5 & 2.401 & 3.00 & 5.00 & 6.00 \\
\hline 25. Estilo de presentación & 5.69 & 6.00 & 5 & 2.057 & 5.00 & 6.00 & 7.00 \\
\hline $\begin{array}{c}\text { 16. FTP (File Transfer } \\
\text { Protocol) }\end{array}$ & 3.45 & 3.00 & 0 & 2.841 & 1.00 & 3.00 & 5.00 \\
\hline $\begin{array}{c}\text { 17. Información en } \\
\text { diferentes formatos }\end{array}$ & 6.01 & 6.00 & 5 & 2.370 & 5.00 & 6.00 & 8.00 \\
\hline $\begin{array}{c}\text { 18. Sintetizar en tablas, } \\
\text { gráficos o esquemas }\end{array}$ & 5.86 & 6.00 & 5 & 2.348 & 4.00 & 6.00 & 8.00 \\
\hline
\end{tabular}




\begin{tabular}{|c|c|c|c|c|c|c|c|}
\hline ITEM & Media & Mediana & Moda & $\begin{array}{l}\text { Desv. } \\
\text { Típica }\end{array}$ & $\begin{array}{l}\text { Perc. } \\
25\end{array}$ & $\begin{array}{l}\text { Perc. } \\
50\end{array}$ & $\begin{array}{c}\text { Perc. } \\
75 \\
\end{array}$ \\
\hline $\begin{array}{l}\text { 19. Organizar con bases de } \\
\text { datos u hojas de calculo }\end{array}$ & 4.91 & 5.00 & 5 & 2.227 & 3.00 & 5.00 & 6.00 \\
\hline 20. Organizadores gráficos & 4.43 & 5.00 & 5 & 2.472 & 3.00 & 5.00 & 6.00 \\
\hline 21. Investigar gracias a las TIC & 6.07 & 6 & 7 & 2.265 & 5 & 6 & 8 \\
\hline $\begin{array}{l}\text { 22. Evaluar autoría y } \\
\text { fiabilidad }\end{array}$ & 5.95 & 6 & 7 & 2.108 & 5 & 6 & 7 \\
\hline $\begin{array}{l}\text { 23. Información en } \\
\text { ordenadores }\end{array}$ & 4.75 & 5.00 & 5 & 2.305 & 3.00 & 5.00 & 6.00 \\
\hline 24. Evaluar multimedia & 4.45 & 5.00 & 5 & 2.245 & 3.00 & 5.00 & 6.00 \\
\hline $\begin{array}{l}\text { 25. Evaluar uso de fuentes } \\
\text { de información y TIC }\end{array}$ & 5.41 & 6.00 & 6 & 2.102 & 4.00 & 6.00 & 7.00 \\
\hline 26. Búsquedas bibliográficas & 5.99 & 6.00 & 5 & 2.098 & 5.00 & 6.00 & 7.00 \\
\hline $\begin{array}{l}\text { 27. Frecuencia de uso: web } \\
\text { de inicio }\end{array}$ & 3.61 & 3.00 & 0 & 3.317 & 1.00 & 3.00 & 6.00 \\
\hline $\begin{array}{l}\text { 28. Frecuencia de uso: } \\
\text { agregadores RSS }\end{array}$ & 1.70 & 1.00 & 0 & 2.196 & 0.00 & 1.00 & 3.00 \\
\hline $\begin{array}{l}\text { 29. Frecuencia de uso: } \\
\text { marcadores sociales }\end{array}$ & 0.77 & 0.00 & 0 & 1.341 & 0.00 & 0.00 & 1.00 \\
\hline $\begin{array}{l}\text { 30. Frecuencia de uso: } \\
\text { enciclopedias online }\end{array}$ & 6.66 & 7.00 & 7 & 1.889 & 5.50 & 7.00 & 8.00 \\
\hline
\end{tabular}

De forma general, en la tabla 3 se observa un alto índice de valoraciones positivas agrupadas (de 5 a 10) entre las que destacan el ítem $\mathrm{n}^{\circ}$ 30 referido a al uso de las Enciclopedias Online (86.6\%), y el ítem 26 sobre competencia en búsqueda bibliográficas (81.8\%).

En el otro extremo nos encontramos con los ítems 28 y 29 sobre frecuencia de uso de agregadores RSS y marcadores sociales, con valores entre 0 y 4 (que indican que nunca se ha usado o una vez al mes) con frecuencias muy altas, $87.2 \%$ y $96.4 \%$ respectivamente, coincidiendo las modas de ambos ítems en el valor 0.

Tabla 4. Frecuencia de respuestas negativas y positivas de los ítems de la Subdimensión INF-A2BC.

\begin{tabular}{ccc}
\hline Item & Negativos (0-4) & Positivos (5-10) \\
\hline 14. Consultas en bases de datos & $48.9 \%$ & $51.1 \%$ \\
\hline 15. Estilo de presentación & $22.8 \%$ & $77.2 \%$ \\
\hline 16. FTP (File Transfer Protocol) & $65.0 \%$ & $35.0 \%$ \\
\hline 17. Información en diferentes formatos & $23.1 \%$ & $76,9 \%$ \\
\hline
\end{tabular}




\begin{tabular}{ccc}
\hline Item & Negativos (0-4) & Positivos (5-10) \\
\hline $\begin{array}{c}\text { 18. Sintetizar en tablas, gráficos o esquemas } \\
\text { 19. Organizar con bases de datos u hojas } \\
\text { de calculo }\end{array}$ & $25.8 \%$ & $74.2 \%$ \\
\hline $\begin{array}{c}\text { 20. Organizadores gráficos } \\
\text { 21. Investigar gracias a las TIC }\end{array}$ & $62.0 \%$ & $62.0 \%$ \\
\hline 22. Evaluar autoría y fiabilidad & $22.1 \%$ & $71.7 \%$ \\
\hline 23. Información en ordenadores & $20.5 \%$ & $79.5 \%$ \\
\hline 24. Evaluar multimedia & $43.2 \%$ & $56.8 \%$ \\
\hline $\begin{array}{c}\text { 25. Evaluar uso de fuentes de información } \\
\text { y TIC }\end{array}$ & $46.8 \%$ & $53.2 \%$ \\
\hline 26. Búsquedas bibliográficas & $30.1 \%$ & $69.9 \%$ \\
\hline 27. Frecuencia de uso: web de inicio & $18.2 \%$ & $81.7 \%$ \\
\hline 28. Frecuencia de uso: agregadores RSS & $87.2 \%$ & $38.3 \%$ \\
\hline 29. Frecuencia de uso: marcadores sociales & $\mathbf{9 6 . 4} \%$ & $3.8 \%$ \\
\hline $\begin{array}{c}\text { 30. Frecuencia de uso: enciclopedias } \\
\text { online }\end{array}$ & $13.4 \%$ & $\mathbf{8 6 . 6} \%$ \\
\hline
\end{tabular}

Si contemplamos de manera holística los datos descriptivos, nos encontramos con que muchos de los ítems presentan altas frecuencias en los valores centrales, y con la moda localizada en el valor central cinco, a excepción del ítem mejor valorado, el número 30 (Enciclopedias online); y los ítems con medidas de tendencia central más bajas, el ítem 16 sobre el uso de protocolos de transferencia de archivos, el ítem 27 sobre la frecuencia de uso de webs de inicio, el ítem 28 sobre la frecuencia de uso de agregadores RSS y el ítem 29 sobre la frecuencia de uso de marcadores sociales, en los que la moda se sitúa en el valor extremo cero.

Por tanto en estos ítems hay un colectivo importante de alumnos que se siente incapaces para utilizar los protocolos de transferencia de archivos (ítem 16) o que nunca ha utilizado ni ha oído hablar de las webs de inicio, agregadores RSS y marcadores sociales (ítems 27, 28 y 29).

\section{Conclusiones}

La mayoría de los futuros maestros, se sienten competentes en cuestiones relacionadas con la navegación por internet, en las referidas a los estilos de presentación, uso de fuentes de información y TIC y en las búsquedas 
bibliográficas; la gran mayoría reconoce utilizar con bastante frecuencia las enciclopedias online. Se muestra el uso habitual de los aspectos más básicos de Internet, tanto en el estudio como en la diversión o los juegos.

Por el contrario, se sienten escasamente competentes en el empleo del FTP (File Transfer Protocol / Protocolo de Transferencia de Archivos) y se autoperciben de forma discretamente competentes en aspectos referidos a la organización de información empleando bases de datos u hojas de cálculo, organización mediante gráficos, gestión de la información en ordenadores y evaluación multimedia. Ello puede explicarse por la poca necesidad de uso que tienen de estas herramientas, por la mayor preparación requerida, y por el nulo uso que hacen de ellas en las clases de la Universidad.

Usan muy poco agregadores RSS y marcadores sociales y, aunque son algo más usadas las webs de inicio, estas resultan serles poco conocidas ya que un $40.2 \%$ afirma que nunca las ha utilizado. En cambio, a pesar de que el almacenamiento virtual es más conocido y usado, casi una quinta parte de los estudiantes encuestados reconocer no haberlos empleado nunca. Ocurre lo mismo que en el caso anterior: no las usan por iniciativa personal, y no se les habitúa al uso en la docencia universitaria o en la investigación.

Estos resultados parecen confirmar los estudios citados en el marco teórico, con los que observamos una fuerte concordancia (Espuny, González y Gisbert, 2010; Roig y Pascual, 2012; Perez y Vilches, 2012; Cabezas, Casillas y Pinto, 2014; Burnett, 2011; Duncan-Howell, 2012; Chen, Zhou, Tan y Wong, 2013; Rokenes y Krumsvik, 2014). Es por tanto, un alumnado con una competencia digital muy superficial, habilidoso, más que competente, en tareas sencillas como la navegación y búsqueda de información en internet, pero sin habilidades complejas en tratamiento de la información ni utilizar recursos útiles para estudiantes como los repositorios virtuales (casi un 20\% nunca lo ha empleado).

Sobre las limitaciones de esta investigación, su aplicación en una única Facultad (Facultad de Educación de León) y el uso de un único instrumento son aspectos a considerar; sin embargo, dada la amplitud de la muestra estos escollos quedan al menos reducidos. Sería enriquecedor realizar investigaciones similares en diferentes localizaciones, así como comparaciones con alumnos recién graduados o profesores en ejercicio.

Para finalizar, este y otros análisis sobre las carencias en competencia digital de los futuros maestros, pueden suponer un reto para nuevas 
fórmulas la formación de los futuros docentes, dada la relevancia de la información y su estrecha relación con las TIC en nuestra sociedad, especialmente si tenemos en cuenta que los avances más allá de la Web 2.0 optimizan la búsqueda y gestión de la información.

Estas cuestiones deben suponer cambios significativos en la formación de los maestros, piedra angular de la Educación de nuestra sociedad.

\section{Referencias bibliográficas}

ABELLA, V.; DELGADO, V. (2015) Aprender a usar twitter y usar twitter para aprender. Profesorado, 19 (1). 364-378. Recuperado de http://goo.gl/gV7QaT

ADELL, J. y CASTAÑEDA, L. (2012). Tecnologías emergentes, ¿pedagogías emergentes?. En J. HERNÁNDEZ, M. PENNESI, D. SOBRINO \& A. VÁZQUEZ (Coords), Tendencias emergentes en educación con TIC (pp. 18-63). Barcelona: Editorial espiral.

ÁLVAREZ, J. F., y GISBERT, M. (2015). Grado de alfabetización informacional del profesorado de Secundaria en España: Creencias y autopercepciones. Comunicar, 45, 187-194. doi: 10.3916/C45-2015-20

AGUADED, J.I. (2012). Apuesta de la ONU por una educación y alfabetización mediáticas [United Nations aiming at Media Literacy Education]. Comunicar, 38, 7-8. doi: 10.3916/C38-2012-01-01

AGUADED, J.I. (2014). Desde la infoxicación al derecho a la comunicación [From Infoxication to the Right to Communicate]. Comunicar, 42, 07-08. doi: doi.org/10.3916/ C42-2014-a1

AREA, M., BORRÁS, J. F., y SAN NICOLÁS, B. (2014). La formación del maestro 2.0: el aprendizaje por tareas en entornos b-learning. Revista Interuniversitaria de Formación del Profesorado, 28 (1), 51-66. Recuperado de http://goo.gl/R0sMvj

AREA, M., y GUARRO, A. (2012). La alfabetización informacional y digital: fundamentos pedagógicos para la enseñanza y el aprendizaje competente. Revista Española de Documentación Científica, n. ${ }^{\circ}$ Monográfico, 46-74.

Recuperado de: http://redc.revistas.csic.es/index.php/redc/article/view/744

BAELO, R. y CANTÓN, I. (2009). Las tecnologías de la información y la comunicación en la educación superior. Estudio descriptivo y de revisión. Revista Iberoamericana de Educación, 50 (7), 1-12.

BURNETT, C. (2011). Preservice teachers' digital literacy practices: exploring contingency in identity and digital literacy in and out of educational contexts. Language and Education, 25 (5), 433-449. doi: 10.1080/09500782.2011.584347

CABERO, J. y BARROSO, J. (2013). Internet aplicado a la educación: webquest, blog y wiki. En J. Barroso Osuna y J. Cabero Almenara (Coords.), Nuevos escenarios digitales: Las tecnologías de la información y la comunicación aplicadas a la formación y desarrollo curricular (pp. 21 - 35). Madrid: Pirámide.

CABERO, J., y BARROSO, J. (2013). La escuela en la sociedad de la información. La 
escuela 2.0. En J. Cabero Almenara, y J. Barroso Osuna (Coord.), Nuevos escenarios digitales (pp. 21-36). Madrid: Pirámide.

CABERO, J. y BARROSO, J. (Coords.) (2015). Nuevos retos en tecnología educativa. Madrid: Editorial Síntesis.

CABERO, J. y LLORENTE, M. C. (2006). La rosa de los vientos. Dominios tecnológicos de las TIC por los estudiantes. Sevilla: GID.

CABEZAS, M., CASILLAS, S. y PINTO, A.M. (2014) Percepción de los alumnos de Educación Primaria de la Universidad de Salamanca sobre su competencia digital. EDUTEC, Revista Electrónica de Tecnología Educativa, 48. Recuperado de: http://www. edutec.es/revista/index.php/edutec-e/article/view/156/21

CANTÓN, I. (2007). Gestión del conocimiento, proceso y competencias. Comunicación y pedagogía: Nuevas tecnologías y recursos didácticos, 218, 15-23.

CANTÓN, I. y BAELO, R. (2011). El profesorado universitario y las tecnologías de la información y de la comunicación (TIC): disponibilidad y formación. Educatio siglo XXI: Revista de la Facultad de Educación, 29, (1), 263-301.

CANTÓN, I. y PINO, M. (Coords.) (2014).Organización de centros educativos en la sociedad del conocimiento. Madrid: Alianza Editorial.

CASTELLS, M. (2001). La galaxia Internet. Reflexiones sobre Internet, Empresa y Sociedad. Madrid: Plaza y Janés.

CHEN, W., ZHOU, X., TAN, A., y WONG, P. S. K. (2013). ICT experiences and competencies of pre- service teachers in the digital age. Recuperado de: https://goo.gl/ BTUiBf

COMISIÓN EUROPEA (2006). Competencias clave para el aprendizaje permanente. Recuperado de: http://eur-lex.europa.eu/legal-content/ES/TXT/?uri=URISERV\%3Ac11090

DE PABLOS PONS, J. (2007). Algunas reflexiones sobre las tecnologías digitales y su impacto a diferentes niveles sociales y educativos. En L. AIRES, J. AZEVEDO, I. GASPAR, I., y A. TEIXEIRA, (Coord.). Comunidades virtuais de aprendizagem e identidades no ensino superior (pp. 31-38). Lisboa: Universida de Aberta.

DE PABLOS PONS, J. (Coord.) (2015). Los centros educativos ante el desafío de las tecnologías digitales. Madrid: La Muralla.

DUNCAN-HOWELL, J. (2012). Digital mismatch: Expectations and realities of digital competency amongst pre-service education students. Australasian Journal of educational Technology, 28 (5), 827-840. Recuperado de: http://www.ascilite.org.au/ajet/ ajet28/duncan-howell.html

ESPUNY, C. y GONZALEZ, J. y GISBERT, M. (2010). ¿Empezamos a navegar? La competencia digital profesional en los futuros maestros. Recuperado de http://www.gabinetecomunicacionyeducacion.com/es/publicaciones/comunicaciones-alfabetizacionmediatica-y-culturas-digitales

FERRARI, A. (2013). DIGCOMP: A Framework for Developing and Understanding Digital Competence in Europe. Luxembourg: Publications Office of the European Union. Recuperado de: http://goo.gl//4t1d8

FERNÁNDEZ, C., CEBREIRO, B., FERNÁNDEZ, C. (2011). Competencias para el aprendizaje en red de los alumnos de educación secundaria en Galicia. Pixel-Bit: Revista de medios y educación, 38, 7-21. 
FERNÁNDEZ, C., CEBREIRO, B. y FERNÁNDEZ, J. C. (2009). Usos de las TIC y preparación de los estudiantes de ciencias de la educación para la sociedad del conocimiento (EDU-CTE). Santiago de Compostela: Universidad de Santiago de Compostela.

GROS, B. (2008). Aprendizajes, conexiones y artefactos. Barcelona: Gedisa.

HERNÁNDEZ PINA, F.; COLÁS BRAVO, M.P.; BUENDÍA EISMAN, L. (Coord.) (2010). Competencias cien-tíficas para la realización de una tesis doctoral. La Coruña: Da Vinci Continental.

INTEF (2014). Marco Común de COMPETENCIA DIGITAL DOCENTE V 2.0. Madrid: Ministerio de Educación, Cultura y Deporte. Recuperado de http://goo.gl/DmZA1t

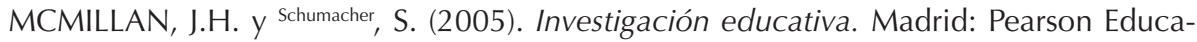
ción.

MARQUÉS, P. (2009). Aportaciones sobre el documento puente: Competencia Digital. Recuperado de: http://goo.gl/QyBzjM

MEC (2007). Las TIC en la Educación. Madrid: MEC.

NONAKA, I. y TAKEUCHI, H. (1999). La organización creadora de conocimiento; como las compañías japonesas crean la dinámica de la innovación. México: Oxford University Press.

PONS, B. y SALINAS, J. (2008). Competències tecnològiques dels alumnes i professors de les Illes Balears. Anuari de l'educació de les Illes Balears. Mallorca: Universitat de les Illes Balears.

PÉREZ, F., y VÍLCHEZ, J. E. (2012). El uso de los videojuegos y redes sociales como predictores de la integración curricular de las TIC en estudiantes de Magisterio. Sphera Pública, 12, 199-215. Recuperado de: http://goo.gl/filurf

PÉREZ, M.A., y DELGADO, A. (2012). De la competencia digital y audiovisual a la competencia mediática: dimensiones e indicadores. Comunicar, 39, 25-34. doi: 10.3916/ C39-2012-02-02

ROBLIZO, M.J, y CÓZAR, R. (2015). Usos y competencias en TIC en los futuros maestros de educación infantil y primaria: Hacia una alfabetización tecnológica real para docentes. Pixel-Bit: Revista de medios y educación, 47, 23-39. doi: 10.12795/pixelbit.2015.i47.02

ROIG, R. y PASCUAL, A. M. (2012). Las competencias digitales de los futuros docentes. Un análisis con estudiantes de Magisterio de Educación Infantil de la Universidad de Alicante. @tic. Revista d'innovació educativa, 9, 53-60 Recuperado de: http://rua. ua.es/dspace/bitstream/10045/25885/1/1473.pdf.

ROKENES, F., y KRUMSVIK, R. J. (2014). Development of Student Teachers' Digital Competence in Teacher Education - A Literature Review. Nordic Journal of Digital Literacy, 04, 250-280. Recuperado de: http://goo.gl/liqOkR

SEVILLANO, M. L. y VÁZQUEZ CANO, E. (2015). Modelos de investigación en contextos ubicuos y móviles en educación superior. Madrid: McGrawHill y UNED

VÁZQUEZ CANO, E. (2013) Microblogging con Edmodo para el desarrollo de las competencias básicas del alumnado de enseñanza secundaria. Un estudio de caso. Educatio Siglo XXI, 1 (31), 313-334. Recuperado de http://revistas.um.es/educatio/article/ view/175191/148331

VIVANCOS, J. (2013). El futuro de la educación y las TIC. Padres y Maestros. Publicación 
Competencia digital y tratamiento de la información en futuros maestros de Primaria Mario Grande-de-Prado, Ruth Cañón-Rodríguez e Isabel Cantón-Mayo

de la Facultad de Ciencias Humanas y Sociales, 351, 22-26. Recuperado de http:// goo.gl/64Ykjz

VIVANCOS, J. (2014). Tratamiento de la información y competencia digital (edición electrónica). Madrid: Alianza Editorial.

ZAFRA, R. (2015). Educación y cultura-red: Potencias y contradicciones para una transformación necesaria. En Profesorado, 2 (19), 1-14. Recuperado de: http://recyt.fecyt. es/index.php/profesorado/article/view/40906/23274 